Tohoku Math. J.

65 (2013), 341-356

\title{
EXTRINSICALLY FLAT MÖBIUS STRIPS ON GIVEN KNOTS IN 3-DIMENSIONAL SPACEFORMS
}

\author{
KosUKE NAOKAWA
}

(Received February 16, 2012, revised October 9, 2012)

\begin{abstract}
We classify the topology of closed extrinsically flat strips which contain a given knot in a 3-dimensional spaceform. This is a refinement and generalization of results of Chicone-Kalton and Røgen.
\end{abstract}

1. Introduction. A $C^{\infty}$-curve in Euclidean 3 -space $\boldsymbol{R}^{3}$ is called regular if its derivative never vanishes. A closed $C^{\infty}$-regular curve $\gamma=\gamma(s): S^{1} \rightarrow \boldsymbol{R}^{3}$ with arc-length parameter $s$ is called a knot if $\gamma$ does not have a self-intersection, where $\boldsymbol{S}^{1}:=\boldsymbol{R} / l \boldsymbol{Z}$, and $l>0$ is the total arc-length of $\gamma$. We fix a knot $\gamma(s)$ arbitrarily, and construct closed strips along $\gamma$, i.e., immersed ruled strips generated by $\gamma$. In particular, we are interested in flat, i.e., zero Gaussian curvature, Möbius strips on such surfaces. A flat and ruled strip is called a developable strip. The topologies of such developable strips are determined by their twisting numbers. Here, the twisting number of a strip takes a half-integer if and only if the strip is non-orientable. If the twisting direction of the strip is clockwise, then its twisting number is positive. The book [13] of Sabitov is useful for references and the history related to developable Möbius strips in $\boldsymbol{R}^{3}$.

The torsion function of a knot $\gamma(s)$ with arc-length parameter $s$ is defined by

$$
\tau(s):=\frac{\operatorname{det}\left(\gamma^{\prime}(s), \gamma^{\prime \prime}(s), \gamma^{\prime \prime \prime}(s)\right)}{\kappa(s)^{2}}
$$

whenever the curvature function $\kappa(s):=\left|\gamma^{\prime \prime}(s)\right|$ does not vanish, where $\gamma^{\prime}:=d \gamma / d s$ and 'det' is the determinant. We have the following.

THEOREM 1.1. Let $\gamma: \boldsymbol{S}^{1} \rightarrow \boldsymbol{R}^{3}$ be a $C^{\omega}$-regular knot, i.e., real-analytic regular $k n o t$, and $Z_{\kappa}$ the zeros of its curvature function $\kappa$. Denote by $\tau(s)$ the torsion function of $\gamma(s)$ defined on $\boldsymbol{S}^{1} \backslash Z_{\kappa}$. Then, the following assertions hold:

(1) Suppose that $\tau(s)$ vanishes identically on $S^{1} \backslash Z_{\kappa}$. Then, a closed developable $C^{\omega}{ }_{-}$ regular (resp. $C^{\infty}$-regular) strip along $\gamma$ can only have zero twisting number.

(2) Suppose that $\tau(s)$ changes sign. Then, for each $n \in(1 / 2) Z$, there exists a closed developable $C^{\omega}$-regular strip along $\gamma$ whose twisting number is $n$.

2010 Mathematics Subject Classification. Primary 53A05.

Key words and phrases. Extrinsically flat surface, Möbius strip, twisting number, Chebyshev space.

Partly supported by the Grant-in-Aid for JSPS Fellows, The Ministry of Education, Culture, Sports, Science and Technology, Japan. 
(3) Suppose that $\tau \leq 0$ on $S^{1} \backslash Z_{\kappa}$ and there exists a point $s_{0} \in S^{1}$ such that $\tau\left(s_{0}\right)<0$. Then, there exists $\lambda_{\gamma} \in(1 / 2) \boldsymbol{Z}$ such that

(i) for each $n \geq \lambda_{\gamma}(n \in(1 / 2) Z)$, there exists a closed developable $C^{\omega}$-regular strip along $\gamma$ whose twisting number is $n$, and

(ii) if $n<\lambda_{\gamma}(n \in(1 / 2) Z)$, there is no closed developable $C^{\infty}$-regular strip along $\gamma$ whose twisting number is $n$.

The assumption $\tau \leq 0$ on $S^{1} \backslash Z_{\kappa}$, or assuming $\tau \geq 0$ instead, in (3) is not essential. In fact, the sign of the torsion function $\tau$ is reversed if one considers the reflection of $\gamma$ across a plane. The invariant $\lambda_{\gamma}$ determined by $\gamma$ in (3) can be written explicitly. In fact, if the zeros $Z_{\kappa}$ of the curvature function $\kappa$ is empty, then $\lambda_{\gamma}$ is equal to the value called the 'self-linking number' of $\gamma$ (see Remark 2.3).

When $Z_{\kappa}$ is empty, the non-existence results (1) and (3)-(ii) have been implicitly proved in Chicone-Kalton [2]. Also, in [2], the existence of a Möbius strip along a given $C^{\omega}$-knot was shown if the twisting number with respect to the Frenet frame is equal to $\pm 1 / 2$. Therefore, the assertions of the theorem are generalizations of the results in [2] to the case that $Z_{\kappa}$ is a non-empty set. In fact, the assumption that $\gamma$ admits a zero of its curvature function is important in the present context. For example, a developable Möbius strip which contains a closed geodesic is called a rectifying Möbius strip. Roughly speaking, a rectifying strip can be constructed from an isometric deformation of a rectangular domain on a plane (cf. [7, Proposition 2.14]). Such a rectifying Möbius strip has at least one zero point of the curvature function of its closed geodesic (cf. [11]). Moreover, the zeros of the curvature function of the geodesic are important when one considers the singularities on the extension of a rectifying Möbius strip. In [9], the author showed that there exist at least three singular points other than cuspidal-edge singularities on the extension of a rectifying Möbius strip. Such a non-cuspidaledge singular point has a special singularity which is 'not a wave front' (e.g. cuspidal cross cap, open swallowtail) if it is on the asymptotic line through a zero curvature point of the closed geodesic.

Røgen [12, Proposition 6] has already proved the existence of a closed developable $C^{\infty}$. regular strip along $\gamma$ whose twisting number is $n \geq \lambda_{\gamma}$ when $\gamma$ is a $C^{\infty}$-knot and satisfies some additional conditions (cf. Proposition 4.5). (His approach is somewhat different from that in Chicone-Kalton [2].) However, if $\gamma$ is real-analytic, then it is natural to expect that the resulting strips can be $C^{\omega}$-regular. In this sense, the above assertions can be considered as a refinement of his assertion. In fact, our assertion is much more difficult to prove. To obtain a $C^{\omega}$-regular developable strip of given twisting number $n \geq \lambda_{\gamma}$, we need a special technique to control the higher order jets when we approximate a $C^{\infty}$-function by Fourier polynomials.

We also consider a generalization of the theorem to the other 3-dimensional spaceforms, i.e., hyperbolic 3-space $\boldsymbol{H}^{3}$ and 3-sphere $\boldsymbol{S}^{3}$. Here, a surface immersed in a spaceform is called extrinsically flat if the product of its two principal curvatures vanishes identically. In this paper, we prove Theorem 1.1 as a corollary of the following assertion: 
THEOREM 1.2. The statement of Theorem 1.1 holds also for extrinsically flat strips in $S^{3}$ (resp. $\left.\boldsymbol{H}^{3}\right)$.

This implies that the condition 'extrinsic flatness' is appropriate for exhibiting its global properties. In fact, Theorem 1.1 follows from the fact that $\boldsymbol{R}^{3}$ can be considered as an affine space in the projective space $\boldsymbol{P}^{3}(\boldsymbol{R})$ and the extrinsically flatness is preserved in this inclusion map. To prove Theorem 1.2, we use a technique which is a refinement of that in ChiconeKalton [2]. Since it is classically known that complete and extrinsically flat regular surfaces in $S^{3}$ are all totally geodesic, the extension of our strip in $S^{3}$ must have singular points. It should be remarked that Honda [5], [6] recently showed the existence of many non-trivial such surfaces with singularities in $\boldsymbol{S}^{3}$ and $\boldsymbol{H}^{3}$.

We also mention the difference between extrinsically flat surfaces and intrinsically flat surfaces in spaceforms. In $\boldsymbol{R}^{3}$, the class of the extrinsically flat surfaces coincides with that of the flat surfaces. On the other hand, it is known that flat surfaces immersed in $\boldsymbol{H}^{3}$ are all orientable (cf. [8]). In particular, there does not exist a flat $C^{\infty}$-regular Möbius strip in $\boldsymbol{H}^{3}$. In $\boldsymbol{S}^{3}$, there are many flat $C^{\infty}$-regular Möbius strips on an arbitrary knot, as shown by Aledo-Gàlvez-Mira [1]. However, Gàlvez-Mira [3] showed that they cannot be real-analytic. This implies that the properties of intrinsically flat surfaces in spaceforms other than $\boldsymbol{R}^{3}$ have different global behaviors from the case of extrinsically flat surfaces.

In Section 2, we give several fundamental definitions. In Section 3, we show that the same discussion as in [2] can be applied for a real-analytic knot whose curvature function might have zero points and that the non-existence assertions (1) and (3)-(ii) of Theorems 1.1 and 1.2 still hold. In Section 4, we prove the existence results (2) and (3)-(i). In Appendix A, we recall Chebyshev spaces and show a lemma with a special technique that is used in the proof of the existence results in Section 4.

2. Preliminaries. We explain Theorem 1.2 for the case $\boldsymbol{S}^{3}$ in detail, and obtain the same assertions for $\boldsymbol{H}^{3}$ and $\boldsymbol{R}^{3}$. As a preliminary, we mention the gnomonic projection of $\boldsymbol{S}^{3}$ and the Klein model of $\boldsymbol{H}^{3}$. We consider the 3-sphere as the unit sphere

$$
\boldsymbol{S}^{3}=\left\{{ }^{t}\left(x_{0}, x_{1}, x_{2}, x_{3}\right) \in \boldsymbol{R}^{4} ; x_{0}^{2}+x_{1}^{2}+x_{2}^{2}+x_{3}^{2}=1\right\}
$$

in $\boldsymbol{R}^{4}$, and set the north hemisphere

$$
S_{+}^{3}:=\left\{{ }^{t}\left(x_{0}, x_{1}, x_{2}, x_{3}\right) \in S^{3} ; x_{0}>0\right\},
$$

where ' $t$ ' denotes the transpose of a vector. A gnomonic projection $G: S_{+}^{3} \rightarrow \boldsymbol{R}^{3}$ is defined by

$$
G(x):={ }^{t}\left(\frac{x_{1}}{x_{0}}, \frac{x_{2}}{x_{0}}, \frac{x_{3}}{x_{0}}\right)
$$

for $x={ }^{t}\left(x_{0}, x_{1}, x_{2}, x_{3}\right) \in S^{3}$. The 'light source' of the gnomonic projection $G$ is the origin of $\boldsymbol{R}^{4}$, and its 'screen' is the hyperplane $x_{0}=1$ in $\boldsymbol{R}^{4}$. The projection $G$ maps a geodesic in $S^{3}$ into a line in $\boldsymbol{R}^{3}$. Moreover, it maps an extrinsically flat surface in $S^{3}$ into a flat surface in $\boldsymbol{R}^{3}$. 
On the other hand, the 'gnomonic projection' for $\boldsymbol{H}^{3}$ can also be considered. The hyperbolic 3 -space $\boldsymbol{H}^{3}$ is regarded as the Minkowski model in the Lorentz-Minkowski space $\boldsymbol{R}_{1}^{4}$, i.e.,

$$
\boldsymbol{H}^{3}=\left\{{ }^{t}\left(x_{0}, x_{1}, x_{2}, x_{3}\right) \in \boldsymbol{R}_{1}^{4} ;\langle x, x\rangle=1, x_{0}>0\right\},
$$

where the Lorentz inner product of $\boldsymbol{R}_{1}^{4}$ is defined by

$$
\langle x, y\rangle:=-x_{0} y_{0}+x_{1} y_{1}+x_{2} y_{2}+x_{3} y_{3}
$$

for $x={ }^{t}\left(x_{0}, x_{1}, x_{2}, x_{3}\right), y={ }^{t}\left(y_{0}, y_{1}, y_{2}, y_{3}\right) \in \boldsymbol{R}_{1}^{4}$. The projection $G: \boldsymbol{H}^{3} \rightarrow \boldsymbol{B}^{3} \subset \boldsymbol{R}^{3}$ defined by the same equality (2.1) maps a geodesic in $\boldsymbol{H}^{3}$ into a line in $\boldsymbol{R}^{3}$, and an extrinsically flat surface in $\boldsymbol{H}^{3}$ into a flat surface in $\boldsymbol{R}^{3}$. Here, $\boldsymbol{B}^{3}$ is the open unit ball whose center is the origin of $\boldsymbol{R}^{3}$. So-called Klein model is the model of $\boldsymbol{H}^{3}$ realized as the open ball $\boldsymbol{B}^{3}$ in $\boldsymbol{R}^{3}$. Consequently, through the two gnomonic projections, $\boldsymbol{H}^{3}$ can be realized in $\boldsymbol{R}^{3}$, and $\boldsymbol{R}^{3}$ can be realized in $S^{3}$. These inclusions say that Theorem 1.2 for $\boldsymbol{H}^{3}$ and Theorem 1.1 follow from Theorem 1.2 for $S^{3}$.

REMARK 2.1. The cases of $\boldsymbol{R}^{3}$ and $\boldsymbol{H}^{3}$ can be also proven directly in the same manner as that of $\boldsymbol{S}^{3}$. In fact, the author showed them individually in the earlier version of this paper. The author appreciates referee's comment that the extrinsic flatness of surfaces in 3dimensional spaceforms is preserved under the gnomonic projections.

From now on, we consider the case of $S^{3}$. Let $\gamma: S^{1} \rightarrow S^{3}$ be a $C^{\omega}$-regular knot. We may assume that $\gamma(s)$ is regarded as the curve $\gamma=\gamma(s): \boldsymbol{R} \rightarrow \boldsymbol{S}^{3}$ having the arc-length parameter $s$ and l-periodicity, i.e., $\gamma(s+l)=\gamma(s)$ for $s \in \boldsymbol{R}$, where $l>0$. The tangent bundle $T S^{3}$ can be regarded as

$$
T_{x} S^{3}=\left\{v \in R^{4} ; x \cdot v=0\right\} \subset R^{4}
$$

for each $x \in S^{3}$, where the dot is the standard inner product in $\boldsymbol{R}^{4}$. We denote by $D$ the LeviCivita connection of $S^{3}$. If the curvature function $\kappa(s):=\left|D_{s} \gamma^{\prime}(s)\right|$ of $\gamma$ does not vanish, then we can take the Frenet frame $\{\boldsymbol{e}, \boldsymbol{n}, \boldsymbol{b}\}$ and the torsion function $\tau$ of $\gamma$, where $|\boldsymbol{v}|:=\sqrt{\boldsymbol{v} \cdot \boldsymbol{v}}$ for $v \in T S^{3}$. In fact,

$$
\boldsymbol{e}:=\gamma^{\prime}, \quad \boldsymbol{n}:=\frac{D_{s} \gamma^{\prime}}{\kappa}, \quad \boldsymbol{b}:=\boldsymbol{e} \times{ }_{\gamma} \boldsymbol{n}, \quad \tau:=-\left(D_{s} \boldsymbol{b}\right) \cdot \boldsymbol{n}
$$

are the unit tangent vector field, the normal vector field, the binormal vector field and the torsion function of $\gamma$, respectively. Here, the cross $\times_{x}: T_{x} S^{3} \times T_{x} S^{3} \rightarrow T_{x} S^{3}$ for each $x \in S^{3}$ is an alternating $(1,2)$-form on $T_{x} S^{3}$ such that

$$
\left(\boldsymbol{v} \times{ }_{x} \boldsymbol{w}\right) \cdot \boldsymbol{c}=\operatorname{det}(x, \boldsymbol{v}, \boldsymbol{w}, \boldsymbol{c})
$$

for $\boldsymbol{v}, \boldsymbol{w}, \boldsymbol{c} \in T_{x} \boldsymbol{S}^{3}$. Although the Frenet frame does not exist on the zeros of $\kappa$, the knot $\gamma$ is now real-analytic, and hence we can define an 'extended' Frenet frame on $\boldsymbol{R}$ as follows: Suppose that there exists a point $s=s_{0}$ such that $\kappa\left(s_{0}\right) \neq 0$. We denote all the zeros of $\kappa(s)$ by

$$
0 \leq s_{1}<s_{2}<\cdots<s_{r}<l .
$$


For each $k=1,2, \ldots, r$, there exist a positive integer $N_{k}$ and a $C^{\omega}$-vector field $\varphi_{k}=\varphi_{k}(s)$ : $I_{k} \rightarrow T S^{3}$ along $\gamma$ such that

$$
\gamma^{\prime}(s) \times_{\gamma(s)} D_{s} \gamma^{\prime}(s)=\left(s-s_{k}\right)^{N_{k}} \varphi_{k}(s) \quad\left(\varphi_{k}\left(s_{k}\right) \neq \mathbf{0}\right)
$$

for $s \in I_{k}$, where $I_{k}$ is an open interval containing $s=s_{k}$. We set

$$
\hat{\boldsymbol{b}}(s):=(-1)^{N_{1}+\cdots+N_{k}} \frac{\varphi_{k}(s)}{\left|\varphi_{k}(s)\right|}
$$

on each $I_{k}$. Then, $\hat{\boldsymbol{b}}(s)$ is equal to either $\boldsymbol{b}(s)$ or $-\boldsymbol{b}(s)$ for $s \in I_{k} \backslash\left\{s_{k}\right\}$. We remark that $\hat{\boldsymbol{b}}(s)$ may change to the opposite direction as it goes around $\gamma$ once. We set

$$
\hat{\boldsymbol{n}}:=\hat{\boldsymbol{b}} \times{ }_{\gamma} \boldsymbol{e}, \quad \hat{\kappa}:=\left(D_{s} \boldsymbol{e}\right) \cdot \hat{\boldsymbol{n}}, \quad \hat{\tau}:=-\left(D_{s} \hat{\boldsymbol{b}}\right) \cdot \hat{\boldsymbol{n}} .
$$

The orthonormal $C^{\omega}$-frame $\{\boldsymbol{e}, \hat{\boldsymbol{n}}, \hat{\boldsymbol{b}}\}$ is called the extended Frenet frame of $\gamma$. Similarly, $\hat{\kappa}$ and $\hat{\tau}$ are called an extended curvature function and an extended torsion function, respectively. Then, the modified Frenet formula

$$
D_{s} \boldsymbol{e}=\hat{\kappa} \hat{\boldsymbol{n}}, \quad D_{s} \hat{\boldsymbol{n}}=-\hat{\kappa} \boldsymbol{e}+\hat{\tau} \hat{\boldsymbol{b}}, \quad D_{s} \hat{\boldsymbol{b}}=-\hat{\tau} \hat{\boldsymbol{n}}
$$

holds. We remark that there exists an orthonormal $C^{\omega}$-frame satisfying the formula (2.2) even if $\kappa$ vanishes everywhere. In this case, we have $\hat{\kappa}=\hat{\tau}=0$. For each point $s$ where $\kappa(s) \neq 0$, we have

$$
\hat{\boldsymbol{n}}(s)=\delta(s) \boldsymbol{n}(s), \quad \hat{\boldsymbol{b}}(s)=\delta(s) \boldsymbol{b}(s),
$$

where $\delta:=\hat{\kappa} / \kappa(= \pm 1)$ is a locally constant function. Although $\hat{\kappa}$ is not equal to $\kappa$, the extended torsion function $\hat{\tau}$ is equal to the torsion function $\tau$ if $\kappa \neq 0$. Therefore, we do not distinguish $\tau$ and $\hat{\tau}$ below and $\hat{\tau}$ is called the torsion function for short.

We explain definitions of closed strips and their twisting numbers. Let $\gamma=\gamma(s): \boldsymbol{R} \rightarrow$ $S^{3}$ be a $C^{\omega}$-regular knot with arc-length parameter $s$, and let $\xi=\xi(s): \boldsymbol{R} \rightarrow T S^{3}$ be a $C^{\omega}$-vector field along $\gamma$. We assume that $\gamma(s)$ is $l$-periodic and $\xi(s)$ is linearly independent of $\gamma^{\prime}(s)$ for each $s \in \boldsymbol{R}$. We define a $C^{\infty}$-map $F_{\gamma, \xi}: \boldsymbol{R} \times(-\varepsilon, \varepsilon) \rightarrow \boldsymbol{S}^{3} \subset \boldsymbol{R}^{4}$ by

$$
F_{\gamma, \xi}(s, u):=\gamma(s) \cos u+\frac{\xi(s)}{|\xi(s)|} \sin u,
$$

where $\varepsilon>0$. Since for each $s=s_{0} \in \boldsymbol{R}$ the curve $u \mapsto F_{\gamma, \xi}\left(s_{0}, u\right)$ is a geodesic in $S^{3}$, the map $F_{\gamma, \xi}$ is a ruled surface. The ruled surface $F_{\gamma, \xi}$ is called a closed strip if $\xi(s)$ is either $l$-periodic, i.e., $\xi(s+l)=\xi(s)$ for $s \in \boldsymbol{R}$, or $l$-odd-periodic, i.e., $\xi(s+l)=-\xi(s)$ for $s \in \boldsymbol{R}$. The knot $\gamma(s)$ is called the generating curve of $F_{\gamma, \xi}$ and $\xi(s)$ is called the ruling vector field of $F_{\gamma, \xi}$. When a positive number $\varepsilon$ is taken sufficiently small, $F_{\gamma, \xi}$ can be a $C^{\omega}$-embedding. We fix such a number $\varepsilon>0$. Let $B$ be the boundary of the image of $F_{\gamma, \xi}$. The number of the connected components of $B$ is one or two. We assign the orientation compatible with $\gamma$ to each connected component of $B$. Then,

$$
\operatorname{Mtn}\left(F_{\gamma, \xi}\right):=\frac{1}{2} \operatorname{Link}(\gamma, B) \in(1 / 2) Z
$$


is called the topological twisting number, or the twisting number for short, of $F_{\gamma, \xi}$, where $\operatorname{Link}(\gamma, B)$ is the sum of linking numbers of all (one or two) connected components of $B$ (cf. [12, Definition 3], [7]).

We normalize the ruling vector $\xi(s)$ for each $s \in \boldsymbol{R}$ such that the projection of $\xi(s)$ into the normal plane at the point $\gamma(s)$ is a unit vector, i.e.,

$$
\left|\boldsymbol{e}(s) \times_{\gamma(s)} \xi(s)\right|=1 \quad(s \in \boldsymbol{R}),
$$

where the normal plane is the plane perpendicular to $\boldsymbol{e}(s)$. The vector field $\xi(s)$ can be expressed as a linear combination of $\{\boldsymbol{e}, \hat{\boldsymbol{n}}, \hat{\boldsymbol{b}}\}$ by

$$
\xi(s)=p(s) \boldsymbol{e}(s)+\cos \theta(s) \hat{\boldsymbol{n}}(s)+\sin \theta(s) \hat{\boldsymbol{b}}(s),
$$

where $p(s)$ and $\theta(s)$ are $C^{\omega}$-functions. Then, there exists an integer $m$ such that

$$
\theta(s+l)=\theta(s)+m \pi .
$$

The number $m / 2$ is called the geometric twisting number of $F_{\gamma, \xi}$ and denoted by $\operatorname{Gtn}\left(F_{\gamma, \xi}\right)$. One can easily check that the topological twisting number and geometric twisting number of $F_{\gamma, \xi}$ satisfy

$$
\operatorname{Mtn}\left(F_{\gamma, \xi}\right)=\operatorname{Mtn}\left(F_{\gamma, \hat{n}}\right)+\operatorname{Gtn}\left(F_{\gamma, \xi}\right) .
$$

The number $\operatorname{Mtn}\left(F_{\gamma, \hat{\boldsymbol{n}}}\right)$ can be regarded as the twisting number of the extended Frenet frame of $\gamma$. Therefore, the geometric twisting number represents the difference between the topological twisting number of the strip $F_{\gamma, \xi}$ and that of the extended Frenet frame.

REMARK 2.2. The number $\operatorname{Mtn}\left(F_{\gamma, \hat{n}}\right)$ coincides with the invariant $\lambda_{\gamma}$ in Theorems 1.1 and 1.2, i.e., $\lambda_{\gamma}=\operatorname{Mtn}\left(F_{\gamma, \hat{n}}\right)$. This is discussed in Sections 3 and 4.

REMARK 2.3. If $\kappa$ never vanishes on $\boldsymbol{R}$, i.e., $Z_{\kappa}=\emptyset$, then the Frenet frame of $\gamma$ can be defined globally, and hence the strip $F_{\gamma, \hat{\boldsymbol{n}}}$ is orientable. Moreover, its twisting number $\operatorname{Mtn}\left(F_{\gamma, \hat{\boldsymbol{n}}}\right)$ is equal to the self-linking number $\operatorname{SL}(\gamma)$. Here, the self-linking number $\operatorname{SL}(\gamma)$ of $\gamma$ is defined by the linking number between $\gamma(s)$ itself and the knot $\gamma(s)+\varepsilon_{0} \boldsymbol{n}(s)\left(s \in \boldsymbol{S}^{1}\right)$, where $\varepsilon_{0}>0$ is sufficiently small ([10], see also [12]). Consequently, by Remark 2.2, if $Z_{\kappa}$ is empty, then $\lambda_{\gamma}$ in Theorems 1.1 and 1.2 is equal to the self-linking number, i.e., $\lambda_{\gamma}=\operatorname{SL}(\gamma)$.

At the end of this section, we formulate a necessary and sufficient condition of the extrinsic flatness of strips by using the expression (2.6). A closed strip $F_{\gamma, \xi}$ is extrinsically developable if and only if $\operatorname{det}\left(\gamma, \gamma^{\prime}, \xi, \xi^{\prime}\right)=0$ on $\boldsymbol{R}$, where $\xi^{\prime}$ is the derivative of $\xi: \boldsymbol{R} \rightarrow T \boldsymbol{S}^{3} \subset \boldsymbol{R}^{4}$ as an $\boldsymbol{R}^{4}$-valued function. By (2.2), the equality

$$
\left(\gamma, \gamma^{\prime}, \xi, \xi^{\prime}\right)=(\gamma, \boldsymbol{e}, \hat{\boldsymbol{n}}, \hat{\boldsymbol{b}})\left(\begin{array}{cccc}
1 & * & * & * \\
0 & 1 & * & * \\
0 & 0 & \cos \theta & p \hat{\kappa}-\left(\theta^{\prime}+\hat{\tau}\right) \sin \theta \\
0 & 0 & \sin \theta & \left(\theta^{\prime}+\hat{\tau}\right) \cos \theta
\end{array}\right)
$$

holds, where the asterisks are certain real numbers. So, the extrinsic flatness is equivalent to

$$
\theta^{\prime}=p \hat{\kappa} \sin \theta-\hat{\tau}
$$


(cf. [2, p. 35]). We remark that the sign of the definition (1.1) of the torsion function of $\gamma$ is opposite to that in [2]

3. Non-existence of extrinsically developable closed strips. Let $\gamma=\gamma(s): \boldsymbol{R} \rightarrow$ $S^{3}$ be a $C^{\omega}$-regular knot. We suppose that the torsion function $\hat{\tau}(s)$ of $\gamma$ is non-negative. Then, we prove that the twisting number of a extrinsically developable closed strip $F_{\gamma, \xi}$ on $\gamma$ must be less than or equal to $\lambda_{\gamma}:=\operatorname{Mtn}\left(F_{\gamma, \hat{\boldsymbol{n}}}\right)$ (remind Remark 2.2). By (2.7), this is equivalent to the geometric twisting number $\operatorname{Gtn}\left(F_{\gamma, \xi}\right)$ being non-positive. If the curvature function of $\gamma$ does not vanish, this assertion follows from the proof of [2, Theorem 2.4]. In this section, we show that a discussion similar to [2] can be applied for an arbitrary $C^{\omega}$-regular knot whose curvature function might vanish.

LEMMA 3.1 ([2]). Let $\gamma(s)$ be a $C^{\omega}$-regular knot in $S^{3}$. If its torsion function $\hat{\tau}$ is non-negative, the geometric twisting number of a closed extrinsically developable $C^{\infty}$-regular strip $F_{\gamma, \xi}$ on $\gamma$ must be non-positive.

Proof. Suppose that the geometric twisting number $\operatorname{Gtn}\left(F_{\gamma, \xi}\right)$ is positive. The ruling vector field $\xi$ is normalized as in (2.5) and expressed by (2.6). Since $\operatorname{Gtn}\left(F_{\gamma, \xi}\right)>0$, the integer $m$ satisfying $\theta(s+l)=\theta(s)+m \pi$ is positive. We may assume $0<\theta(0) \leq \pi$ by replacing $\xi(0)$ with $-\xi(0)$, if necessary. Moreover, we suppose $0<\theta(0)<\pi$ by a parallel shift of the parameter $s$. Since $\theta(l)=\theta(0)+m \pi>\pi$, we take the first point $s=s_{1} \in(0, l)$ where $\theta(s)$ is equal to $\pi$, and choose a point $s=s_{0}$ such that $0<\theta(s)<\pi$ for $s \in\left[s_{0}, s_{1}\right)$. Then, for each $s \in\left[s_{0}, s_{1}\right)$, we have

$$
\begin{aligned}
\int_{s_{0}}^{s} p(s) \hat{\kappa}(s) d s & =\int_{s_{0}}^{s} \frac{\theta^{\prime}(s)+\hat{\tau}(s)}{\sin \theta(s)} d s \geq \int_{s_{0}}^{s} \frac{\theta^{\prime}(s)}{\sin \theta(s)} d s \\
& =\log \left(\tan \frac{\theta(s)}{2}\right)-\log \left(\tan \frac{\theta\left(s_{0}\right)}{2}\right)
\end{aligned}
$$

by (2.8). Therefore, when $s$ approaches $s_{1}$ from below, this left-hand side converges to a finite value, but this right-hand side diverges to positive infinity. This is a contradiction.

4. Constructions of extrinsically developable closed strips. We suppose that the torsion function of a given $C^{\omega}$-regular $\operatorname{knot} \gamma(s)$ has a negative value. Let $\{\boldsymbol{e}, \hat{\boldsymbol{n}}, \hat{\boldsymbol{b}}\}$ be the extended Frenet frame, $\hat{\kappa}$ the extended curvature function and $\hat{\tau}$ the torsion function of $\gamma$. Then, we prove that for each $n \geq \lambda_{\gamma}:=\operatorname{Mtn}\left(F_{\gamma, \hat{n}}\right)(n \in(1 / 2) Z)$, there exists an extrinsically developable $C^{\omega}$-regular strip $F_{\gamma, \xi}$ along $\gamma$ such that its topological twisting number $\operatorname{Mtn}\left(F_{\gamma, \xi}\right)$ is equal to $n$ (remind Remark 2.2). By (2.7), this is equivalent to constructing an extrinsically developable $C^{\omega}$-regular strip $F_{\gamma, \xi}$ such that its geometric twisting number $\operatorname{Gtn}\left(F_{\gamma, \xi}\right)$ is equal to an arbitrary non-negative number $m / 2(m \in \boldsymbol{Z})$.

We fix an arbitrary non-negative integer $m$. We set

$$
\Theta^{\omega}:=\left\{\theta \in \mathcal{C}^{\omega}(\boldsymbol{R}) ; \theta(s+l)=\theta(s)+m \pi \quad(s \in \boldsymbol{R})\right\},
$$

where $\mathcal{C}^{\omega}(\boldsymbol{R})$ is the set of the $C^{\omega}$-functions on $\boldsymbol{R}$. We would like to take an appropriate $C^{\omega}$ function $\theta(s) \in \Theta^{\omega}$ such that the equation (2.8) is satisfied for a certain $C^{\omega}$-function $p(s)$ on 
$\boldsymbol{R}$. If the vector field $\xi(s)$ is defined by (2.6), the closed strip $F_{\gamma, \xi}$ is extrinsically developable. We suppose that $\theta \in \Theta^{\omega}$ is taken in an appropriate manner. If $\hat{\kappa}(s) \sin \theta(s)$ does not vanish, such a function $p(s)$ is determined by

$$
p(s):=\frac{\theta^{\prime}(s)+\hat{\tau}(s)}{\hat{\kappa}(s) \sin \theta(s)} .
$$

Then, the extrinsically flat condition (2.8) holds. Therefore, we wish to choose a function $\theta \in \Theta^{\omega}$ so that the right-hand side of (4.1) converges to a finite number when $\hat{\kappa}(s) \sin \theta(s)$ approaches zero. If $s=s_{0}$ is a zero point of $\hat{\kappa}(s)$ (resp. $\hat{\tau}(s)$ ), then we denote its multiplicity (see Appendix A) by $N$ (resp. $M$ ). If $s=s_{0}$ is not a zero point of $\hat{\kappa}(s)($ resp. $\hat{\tau}(s)$ ), then we define $N=0$ (resp. $M=0$ ).

We would like to take a function $\theta \in \Theta^{\omega}$ such that the following conditions (A) and (B) are satisfied at any point $s=s_{0}$ where $\hat{\kappa}(s) \sin \theta(s)$ vanishes:

(A) If $\theta\left(s_{0}\right)$ is not an integer multiple of $\pi$, then

$$
\theta^{(k+1)}\left(s_{0}\right)=-\hat{\tau}^{(k)}\left(s_{0}\right) \quad(0 \leq k \leq N-1),
$$

(B) if $\theta\left(s_{0}\right)$ is an integer multiple of $\pi$, then

$$
\begin{aligned}
& \left.\theta^{\prime}\left(s_{0}\right)=\cdots=\theta^{(M)}\left(s_{0}\right)=0 \quad \text { (if } M>0\right), \\
& \theta^{(M+1)}\left(s_{0}\right)=-\hat{\tau}^{(M)}\left(s_{0}\right), \ldots, \theta^{(M+N+1)}\left(s_{0}\right)=-\hat{\tau}^{(M+N)}\left(s_{0}\right) .
\end{aligned}
$$

When $\theta \in \Theta^{\omega}$ satisfies these conditions, $p(s)$ is real-analytic on $\boldsymbol{R}$ and satisfies (2.8). In fact, suppose that $\hat{\kappa}(s) \sin \theta(s)$ vanishes at $s=s_{0}$. In the case that $\theta\left(s_{0}\right)$ is not an integer multiple of $\pi$, i.e., in the case of the condition (A), we obtain

$$
\theta^{(k+1)}\left(s_{0}\right)+\hat{\tau}^{(k)}\left(s_{0}\right)=\hat{\kappa}^{(k)}\left(s_{0}\right)=0 \quad(0 \leq k \leq N-1)
$$

and $\hat{\kappa}^{(N)}\left(s_{0}\right) \neq 0$. Since we have

$$
\lim _{s \rightarrow s_{0}} \frac{\theta^{\prime}(s)+\hat{\kappa}(s) \sin \theta(s)}{\hat{\kappa}(s)}=\frac{1}{\sin \theta\left(s_{0}\right)} \frac{\theta^{(N+1)}\left(s_{0}\right)+\hat{\tau}^{(N)}\left(s_{0}\right)}{\hat{\kappa}^{(N)}\left(s_{0}\right)},
$$

$p(s)$ defined by (4.1) has the limit as $s$ tends to $s_{0}$. Hence, $p(s)$ is real-analytic at $s=s_{0}$. On the other hand, when $\theta\left(s_{0}\right)$ is an integer multiple of $\pi$, i.e., in the case of the condition (B), we obtain

$$
\left.\frac{d^{k}(\hat{\kappa} \sin \theta)}{d s^{k}}\right|_{s=s_{0}}=0 \quad(0 \leq k \leq M+N)
$$

and

$$
\begin{aligned}
\left.\frac{d^{M+N+1}(\hat{\kappa} \sin \theta)}{d s^{M+N+1}}\right|_{s=s_{0}} & =\left.\left(\begin{array}{c}
M+N+1 \\
N
\end{array}\right) \hat{\kappa}^{(N)}(s)(\sin \theta(s))^{(M+1)}\right|_{s=s_{0}} \\
& =-\cos \theta\left(s_{0}\right)\left(\begin{array}{c}
M+N+1 \\
N
\end{array}\right) \hat{\kappa}^{(N)}\left(s_{0}\right) \hat{\tau}^{(M)}\left(s_{0}\right) \neq 0 .
\end{aligned}
$$

Therefore, the limit as $s$ tends to $s_{0}$ of $p(s)$ exists, so $p(s)$ is real-analytic at $s=s_{0}$. Considering the above, we prove the following assertion. 
LemMa 4.1. Let $\gamma(s)$ be a $C^{\omega}$-regular knot in $S^{3}$. If its torsion function $\hat{\tau}(s)$ has a negative value, then for each non-negative integer $m$, there exists a closed extrinsically developable $C^{\omega}$-regular strip on $\gamma$ whose geometric twisting number is equal to $m / 2$.

PROOF. Let $s=s_{0}$ be a negative value of $\hat{\tau}(s)$. We denote by $t_{1}, t_{2}, \ldots, t_{r} \in[0, l)$ all the zeros of the extended curvature function $\hat{\kappa}(s)$ of $\gamma$ and by $N_{1}, N_{2}, \ldots, N_{r}$ their multiplicities. We may sort them so that $t_{1}<t_{2}<\cdots<t_{r}$. Moreover, since $\kappa(s)$ does not vanish at $s=s_{0}$, we may assume $0<t_{1}<t_{2}<\cdots<t_{r}<s_{0}<l$ by a parallel shift of the parameter $s$, if necessary. We take a sufficiently small open interval neighborhood $(a, b)$ of $s=s_{0}$, such that $\hat{\tau}(s)$ is negative on $(a, b)$ and the interval $(a, b)$ contains none of $s=t_{1}, t_{2}, \ldots, t_{r}$ nor $s=l$. We choose $m$ arbitrary points from $(a, b)$ and denote these points by $s_{1}, s_{2}, \ldots, s_{m}$ $\left(a<s_{1}<s_{2}<\cdots<s_{m}<b\right)$. We would like to take a function $\theta(s) \in \Theta^{\omega}$ with the following properties:

(1) $\theta(s+l)=\theta(s)+m \pi$ for $s \in \boldsymbol{R}$,

(2) $0<\theta\left(t_{h}\right)<\pi$ and $\theta^{(k+1)}\left(t_{h}\right)=-\hat{\tau}^{(k)}\left(t_{h}\right)$ for each $1 \leq h \leq r$ and each $1 \leq k \leq$ $N_{h}-1$,

(3) $\theta\left(s_{h}\right)=h \pi, \theta^{\prime}\left(s_{h}\right)=-\hat{\tau}\left(s_{h}\right)$ for each $1 \leq h \leq m$,

(4) $0<\theta(s)<\pi$ for $0 \leq s \leq a$, and $m \pi<\theta(s)<(m+1) \pi$ for $b \leq s \leq l$,

(5) $\theta^{\prime}(s)>0$ for $a<s<b$.

If we can find such a function $\theta(s)$, the points where $\theta(s)$ are integer multiples of $\pi$ in $[0, l)$ are only the $m$ points $s=s_{1}, \ldots, s_{m}$ by (3), (4) and (5). Therefore, the vanishing points of $\hat{\kappa}(s) \sin \theta(s)$ are only $s=t_{1}, \ldots, t_{r}$ and $s=s_{1}, \ldots, s_{m}$. By (2), at $s=t_{h}(1 \leq h \leq r)$, the condition (A) is satisfied ( $N=N_{h}$ in this case). Also, by (3), at $s=s_{h}(1 \leq h \leq m)$, the condition (B) is satisfied ( $N=M=0$ in this case). Hence, we can conclude the proof if such a function $\theta(s)$ is found.

Let us construct $\theta(s) \in \Theta^{\omega}$ such that (1) through (5) holds. Now, there obviously exists a function $\theta(s)$ satisfying (1) through (5) such that it is of class $C^{\infty}$. We desire, however, a real-analytic one. We denote by $\tilde{\theta}(s)$ such a $C^{\infty}$-function $\theta(s)$. Then, we set

$$
\varphi(s):=\tilde{\theta}(s)-\frac{m \pi}{l} s .
$$

The $C^{\infty}$-function $\varphi(s)$ is $l$-periodic. We replace $s_{1}, \ldots, s_{m}$ with $t_{r+1}, \ldots, t_{r+m}$ and put

$$
v_{1}:=N_{1}+1, \quad v_{2}:=N_{2}+1, \ldots, v_{r}:=N_{r}+1, \quad v_{r+1}:=v_{r+2}:=\cdots:=v_{r+m}:=2 .
$$

We apply Lemma A.4 for the $C^{\infty}$-function $\varphi(s)$, the points $t_{1}, \ldots, t_{r+m}$ and the positive integers $v_{1}, \ldots, v_{r+m}$. Then, we obtain a sequence $\left\{\varphi_{q}\right\}_{q=1}^{\infty}$ of $l$-periodic $C^{\omega}$-functions satisfying

$$
\varphi_{q}^{(k)}\left(t_{h}\right)=\varphi^{(k)}\left(t_{h}\right) \quad\left(1 \leq k \leq N_{h}\right)
$$

for each $1 \leq h \leq r$ and

$$
\varphi_{q}\left(s_{h}\right)=\varphi\left(s_{h}\right), \quad \varphi_{q}^{\prime}\left(s_{h}\right)=\varphi^{\prime}\left(s_{h}\right)
$$


for each $1 \leq h \leq m$, such that $\left\{\varphi_{q}\right\}$ converges uniformly to $\varphi$ on $\boldsymbol{R}$ and $\left\{\varphi_{q}^{\prime}\right\}$ also converges uniformly to $\varphi^{\prime}$. For each $q \in \boldsymbol{N}$, a $C^{\omega}$-function $\theta_{q}$ on $\boldsymbol{R}$ is defined by

$$
\theta_{q}(s):=\varphi_{q}(s)+\frac{m \pi}{l} s,
$$

and then $\theta=\theta_{q}$ satisfies (1) through (3). Moreover, since $\theta_{q}$ and $\theta_{q}^{\prime}$ converge uniformly to $\tilde{\theta}$ and $\tilde{\theta}^{\prime}$, respectively, we can take a sufficient large natural number $q=q_{0}$ such that $\theta=\theta_{q_{0}}$ also satisfies (4) and (5). We can construct $\theta=\theta_{q_{0}} \in \Theta^{\omega}$ satisfying the conditions (A) and (B). Therefore, the assertion holds.

REMARK 4.2. A technique to construct a $C^{\omega}$-function from a $C^{\infty}$-function, while keeping some of the given properties (1) through (5) in the proof of Lemma 4.1, is a key point of this proof. Although a similar technique is used in Kurono-Umehara [7, Proposition 4.3], Lemma A.4 of Appendix A is a generalization of their discussion.

PROOF OF THEOREMS 1.1 AND 1.2. Since the extrinsic flatness of surfaces in spaceforms is preserved under gnomonic projections, the cases of $\boldsymbol{H}^{3}$ and $\boldsymbol{R}^{3}$ follow from that of $\boldsymbol{S}^{3}$ (see Remark 2.1). For a given knot $\gamma$, we set $\lambda_{\gamma}:=\operatorname{Mtn}\left(F_{\gamma, \hat{\boldsymbol{n}}}\right)$. Then, by (2.7), the topological twisting number $\operatorname{Mtn}\left(F_{\gamma, \xi}\right)$ of a close strip $F_{\gamma, \xi}$ is equal to $\lambda_{\gamma}$ if and only if the geometric twisting number $\operatorname{Gtn}\left(F_{\gamma, \xi}\right)$ is equal to zero. So, Lemma 3.1 implies (1) and (3)-(ii) of Theorems 1.1 and 1.2. Also, Lemma 4.1 implies (2) and (3)-(i). We obtain the assertions.

We show two examples of flat strips along $\gamma$ in $\boldsymbol{R}^{3}$.

EXAMPLE 4.3 ([15]). The map $F_{\gamma, \xi}(t, u)=\gamma(t)+u \xi(t)$ defined by

$$
\gamma(t):=\frac{1}{9+4 t^{2}+4 t^{4}+t^{6}}\left(\begin{array}{c}
9 t+6 t^{3}+3 t^{5} \\
12 t+6 t^{3} \\
-72 / 5
\end{array}\right), \quad \xi(t):=\frac{\tau(t)}{\kappa(t)} \boldsymbol{e}(t)+\boldsymbol{b}(t)
$$

for $t \in \boldsymbol{R} \cup\{\infty\}$ gives a rectifying Möbius strip, where $\kappa, \tau, \boldsymbol{e}$ and $\boldsymbol{b}$ are the curvature function, the torsion function, the unit tangent vector and the binormal vector of $\gamma$, respectively. The zeros of $\kappa$ is only $t=\infty$. Since $\tau(0)$ is positive and $\tau(1)$ is negative, there exists a developable closed strip containing $\gamma$ whose topological twisting number is an arbitrary number $n \in(1 / 2) \boldsymbol{Z}$ by (2) of Theorem 1.1.

EXAMPLE 4.4. We consider the regular curve

$$
\gamma(t):=\frac{1}{5}\left(\begin{array}{c}
5 \cos t-\cos t \cos 5 t \\
5 \sin t-\sin t \cos 5 t \\
\sin 5 t
\end{array}\right) \quad(0 \leq t<2 \pi),
$$

which twists five times per turn on a torus. We can prove that the curvature function and the torsion function of $\gamma$ are both positive by using interval arithmetics. We have $\lambda_{\gamma}=$ $\operatorname{Mtn}\left(F_{\gamma, \boldsymbol{n}}\right)=5$, where $\boldsymbol{n}$ is the normal vector field of $\gamma$. Hence, there exists a developable 
closed strip containing $\gamma$ whose topological twisting number is less than or equal to five, but there does not exist one whose topological twisting number is greater than five.

When $\gamma$ is a $C^{\infty}$-regular knot in $\boldsymbol{R}^{3}$, an assertion similar to Theorem 1.1 was proven by Røgen [12]. Strictly speaking, he classified the topologies of developable closed strips along a given $C^{\infty}$-knot $\gamma$ in $\boldsymbol{R}^{3}$ satisfying the following conditions; the curvature function $\kappa$ of $\gamma$ vanishes only on a union of finite numbers of points and finite numbers of intervals, and the torsion function of $\gamma$ converges to zero whenever $\kappa$ approaches zero. His result can be generalized for 3-dimensional spaceforms as follows:

PROPOSITION 4.5. Theorem 1.2 is also true for a $C^{\infty}$-regular knot $\gamma$ satisfying the above conditions. The constructed closed strip corresponding to (2) and (3)-(i) in Theorem 1.2 is of class $C^{\infty}$.

It suffices to prove the assertion in the case of $\boldsymbol{S}^{3}$. Let $\gamma=\gamma(s): \boldsymbol{S}^{1} \rightarrow \boldsymbol{S}^{3} \subset \boldsymbol{R}^{4}$ be a $C^{\infty}$-regular knot with the above conditions. Suppose that there exists a point $s=s_{0}$ where the curvature function $\kappa(s)$ does not vanish and the torsion function $\tau(s)$ is negative. Then, an extrinsically developable closed strip along $\gamma$ is constructed by two parts, i.e., a twisting part and the rest of the strip, as follows:

(a) We take a sufficiently small open interval $I=\left(s_{0}-\delta, s_{0}+\delta\right)(\delta>0)$, on which $\kappa(s)$ does not vanish and $\tau(s)$ is negative. Let $n \in(1 / 2) \boldsymbol{Z}$ be an arbitrary nonnegative number. By the discussions of Theorems 1.1 and 1.2, there exists a (nonclosed) extrinsically developable $C^{\infty}$-regular strip along $\gamma(I)$ such that the 'twisting number' is equal to $n$. Here, the 'twisting number' is the number

$$
\frac{1}{2 \pi}\left(\theta\left(s_{0}+\delta\right)-\theta\left(s_{0}-\delta\right)\right),
$$

where $\theta(s)$ is defined by (2.6) for the Frenet frame $\{\boldsymbol{e}, \boldsymbol{n}, \boldsymbol{b}\}$ of $\gamma$.

(b) An extrinsically developable strip on the remainder $\gamma\left(S^{1} \backslash I\right)$ is constructed as follow: Let $V$ be a unit normal $C^{\infty}$-vector field on $S^{1} \backslash I$ which is parallel with respect to the normal connection of $\gamma$, i.e., $D_{s} V(s)$ is linearly dependent of the unit tangent vector $\boldsymbol{e}(s)$ at each point. Here, $D$ is the Levi-Civita connection of $\boldsymbol{S}^{3}$. For an arbitrary fixed point $s=s_{1} \in S^{1} \backslash I$, the vector field $V$ is determined by the initial value $V\left(s_{1}\right)$. Then, the strip $F_{\gamma, V}:\left(S^{1} \backslash I\right) \times(-\varepsilon, \varepsilon) \rightarrow S^{3} \subset \boldsymbol{R}^{4}$ defined by

$$
F_{\gamma, V}(s, u):=\gamma(s) \cos u+V(s) \sin u \quad\left(s \in S^{1} \backslash I,|u|<\varepsilon\right)
$$

is extrinsically developable, where $\varepsilon>0$ is sufficiently small.

Although the ruling vectors at both ends of these two strips do not coincide in general, the two strips can be joined smoothly by the following lemma:

LEMMA 4.6. Let $J=[a, d], J_{1}:=[a, c]$ and $J_{2}:=[b, d](a<b<c<d)$ be closed intervals, and $\gamma: J \rightarrow S^{3} \subset \boldsymbol{R}^{4}$ a $C^{\infty}$-regular space curve. Suppose that two $C^{\infty}$-regular extrinsically developable strips $F_{\gamma}\left(J_{k}\right)$, $\xi_{k}$ defined by $(2.3)$ along $\gamma\left(J_{k}\right)(k=1,2)$ satisfy

(1) the curvature function $\kappa$ of $\gamma$ does not vanish on $\gamma\left(J_{1} \cap J_{2}\right)$, 
(2) the ruling vectors $\xi_{k}(s)(k=1,2)$ do not contain the osculating plane of $\gamma(s)$ for each $s \in J_{1} \cap J_{2}$, where the osculating plane is the plane spanned by the tangent vector and the principal normal vector of $\gamma$.

Then, there exists a $C^{\infty}$-regular extrinsically developable strip $F_{\gamma, \xi}$ satisfying either

$$
\xi(s)=\left\{\begin{array}{ll}
\xi_{1}(s) & \left(s \in J_{1} \backslash J_{2}\right), \\
\xi_{2}(s) & \left(s \in J_{2} \backslash J_{1}\right),
\end{array} \quad \text { or } \quad \xi(s)= \begin{cases}\xi_{1}(s) & \left(s \in J_{1} \backslash J_{2}\right) \\
-\xi_{2}(s) & \left(s \in J_{2} \backslash J_{1}\right)\end{cases}\right.
$$

To apply this lemma, we must slightly enlarge the intervals $I$ and $S^{1} \backslash I$ and denote them by $J_{1}$ and $J_{2}$. Moreover, to satisfy the above two conditions (1) and (2), the strip $F_{\gamma\left(S^{1} \backslash I\right), V}$ can be deformed a little by changing the initial value $V\left(s_{1}\right)$, if necessary.

Proof OF Lemma 4.6. On $J_{1} \cap J_{2}$ the two ruling vector fields $\xi_{k}(k=1,2)$ can be expressed by the linear combination of the Frenet frame $\{\boldsymbol{e}, \boldsymbol{n}, \boldsymbol{b}\}$ of $\gamma$ as

$$
\xi_{k}(s)=p_{k}(s) \boldsymbol{e}(s)+\cos \theta_{k}(s) \boldsymbol{n}(s)+\sin \theta_{k}(s) \boldsymbol{b}(s) \quad\left(s \in J_{1} \cap J_{2}\right) .
$$

We may assume that $0<\theta_{1}(s), \theta_{2}(s)<\pi\left(s \in J_{1} \cap J_{2}\right)$ by reversing the direction of $\xi_{2}(s)$, if necessary. Let $\left\{\varphi_{1}, \varphi_{2}\right\}$ be a partition of unity subordinate to $\left\{J_{1}, J_{2}\right\}$. We set the $C^{\infty}$-function

$$
\theta(s):=\varphi_{1}(s) \theta_{1}(s)+\varphi_{2}(s) \theta_{2}(s) \quad\left(s \in J_{1} \cap J_{2}\right) .
$$

For $s \in J_{1} \cap J_{2}$, the functions $p(s)$ and $\xi(s)$ are defined as in (4.1) and (2.6), respectively. Then, $F_{\gamma, \xi}$ is extrinsically developable.

PROOF OF PROPOSITION 4.5. If the torsion function $\tau$ of $\gamma$ takes both sign, a constructed closed strip on $\gamma$ is divided into a part of $\tau<0$, a part of $\tau>0$ and the rest of the strip. For example, in the part of $\tau<0$, we can construct a developable strip along $\gamma$ so that it twists clockwise across the osculating plane of $\gamma$. By the above discussion (a), (b) and Lemma 4.6, we obtain the assertion corresponding to (2) and (3)-(i). On the other hand, suppose that $\tau$ is non-positive on $S^{1} \backslash Z_{\kappa}$, where $Z_{\kappa}$ is the zero of $\kappa$. By Lemma 3.1, any extrinsically developable strip along $\gamma\left(S^{1} \backslash Z_{K}\right)$ can not be twisted anti-clockwise across the osculating planes of $\gamma$. Moreover, such a strip along $\gamma\left(Z_{\kappa}\right)$ is contained in totally geodesic surfaces in $S^{3}$. Since the limit of the principal normal vector $\boldsymbol{n}(s)$ of $\gamma$ as $s$ tends to the boundary of $S^{1} \backslash Z_{\kappa}$ exists, (1) and (3)-(ii) hold.

Finally, in Theorems 1.1 and 1.2, we consider the case that finitely many ruling vectors are arbitrarily prescribed. An extrinsically flat strip along $\gamma$ having the prescribed ruling vectors can also be constructed as well as that of the theorems. The number $\lambda_{\gamma}$, determined by $\gamma$, does not change under generic choices of the ruling vectors, and the following assertion holds:

COROllary 4.7. Let $X^{3}$ be a 3-dimensional spaceform, i.e., $X^{3}=S^{3}, \boldsymbol{R}^{3}$ or $\boldsymbol{H}^{3}$, and $\gamma=\gamma(s): S^{1} \rightarrow X^{3}$ a $C^{\omega}$-regular knot whose torsion function $\hat{\tau}$ has a negative value. Take finite points $c_{1}, c_{2}, \ldots, c_{h} \in S^{1}$ and tangent vectors $\left\{\xi_{k} \in T_{\gamma\left(c_{k}\right)} X^{3}\right\}_{k=1}^{h}$. Suppose that for each $k=1,2, \ldots, h$, the vector $\xi_{k}$ is not parallel to $\gamma^{\prime}\left(c_{k}\right)$ and is not perpendicular to $\hat{\boldsymbol{b}}\left(c_{k}\right)$. Then, for each $n \in(1 / 2) \boldsymbol{Z}$ satisfying $n \geq \lambda_{\gamma}:=\operatorname{Mtn}\left(F_{\gamma, \hat{\boldsymbol{n}}}\right)$, there 
exists a closed extrinsically developable $C^{\omega}$-regular strip $F_{\gamma, \xi}$ on $\gamma$ such that $\xi\left(c_{k}\right) \in\left\{ \pm \xi_{k}\right\}$ $(k=0,1,2, \ldots, h)$ and $\operatorname{Mtn}\left(F_{\gamma, \xi}\right)=n$.

Proof. For each $k=1,2, \ldots, h$, we may normalize $\xi_{k}$ so that $\left|\boldsymbol{e}\left(c_{k}\right) \times_{\gamma}\left(c_{k}\right) \xi_{k}\right|=1$. If necessary, the direction of $\xi_{k}$ is reversed. Then, $\xi_{k}$ can be expressed by

$$
\xi_{k}=p_{k} \boldsymbol{e}\left(c_{k}\right)+\cos \theta_{k} \hat{\boldsymbol{n}}\left(c_{k}\right)+\sin \theta_{k} \hat{\boldsymbol{b}}\left(c_{k}\right) \quad\left(p_{k} \in \boldsymbol{R}, \theta_{k} \in[0, \pi)\right) .
$$

Since $\xi_{k}$ is not perpendicular to $\hat{\boldsymbol{b}}\left(c_{k}\right)$, we have $\theta_{k} \neq 0$. We take an open interval $(a, b) \subset$ $(0, l)$ which contains neither $c_{1}, \ldots, c_{h}$ nor the zeros $t_{1}, \ldots, t_{r}$ of $\kappa$. Without loss of generality, we may assume that $0<c_{1}, \ldots, c_{h}, t_{1}, \ldots, t_{r}<a$ is satisfied. Let $s_{1}<\cdots<s_{n}$ be $n$ points in $(a, b)$. By Lemma A.4, we can take a $C^{\omega}$-function $\theta \in \Theta^{\omega}$ satisfying the properties (1) through (5) in the proof of Lemma 4.1, and the following properties (6), (7):

(6) $\theta\left(c_{k}\right)=\theta_{k}(k=1,2, \ldots, h)$,

(7) $\lim _{s \rightarrow c_{k}} p(s)=p_{k}(k=1,2, \ldots, h)$, where $p(s)$ is defined by (4.1).

In fact, the property (7) is equivalent that $\theta^{\left(\tilde{N}_{k}+1\right)}\left(c_{k}\right)$ satisfies

$$
\theta^{\left(\tilde{N}_{k}+1\right)}\left(c_{k}\right)=p_{k} \kappa^{\left(\tilde{N}_{k}\right)}\left(c_{k}\right) \sin \theta_{k}-\hat{\tau}^{\left(\tilde{N}_{k}\right)}\left(c_{k}\right),
$$

where $\tilde{N}_{k}$ is the multiplicity of the zero point $s=c_{k}$ of $\hat{\kappa}$ if $\hat{\kappa}\left(c_{k}\right)=0$ (resp. $\tilde{N}_{k}=0$ if $\hat{\kappa}\left(c_{k}\right) \neq 0$ ). By Lemma A.4, there exists $\left\{\theta_{q}\right\}_{q=1}^{\infty} \subset \Phi^{\omega}$ such that $\theta_{q}$ satisfies (1) through (3), (6) and (7) for each positive integer $q$, and $\theta_{q_{0}}$ satisfies (4) and (5) for a sufficiently large positive integer $q=q_{0}$. If we set $\theta:=\theta_{q_{0}}$, then the closed strip $F_{\gamma, \xi}$ defined by (2.3) and (2.6) is a $C^{\omega}$-regular extrinsically developable strip and has a required twisting number. Moreover, its ruling vector field $\xi(s)$ is parallel to $\xi_{k}$ at $s=c_{k}$ for each $k=1,2, \ldots, h$.

We remark the condition " $\xi_{k}$ is not perpendicular to $\hat{\boldsymbol{b}}\left(c_{k}\right)$ " is not essential. In fact, even if $\theta_{k}=0$ for some $k=1,2, \ldots, h$, we can construct a $C^{\omega}$-regular extrinsically developable strip so that $\xi\left(c_{k}\right) \in\left\{ \pm \xi_{k}\right\}(k=0,1,2, \ldots, h)$. However, the number $\lambda_{\gamma}$ might depend on the choices of $c_{k}$ and $\xi_{k}$.

Appendix A. Chebyshev spaces. We recall Chebyshev spaces (cf. ThorbergssonUmehara [14]). Let $\mathcal{C}^{\infty}(\boldsymbol{R})$ be the function space of class $C^{\infty}$ on $\boldsymbol{R}$. If a zero $t=t_{0}$ of $\varphi(t) \in \mathcal{C}^{\infty}(\boldsymbol{R})$ satisfies

$$
\varphi\left(t_{0}\right)=\varphi^{\prime}\left(t_{0}\right)=\cdots=\varphi^{(\mu-1)}\left(t_{0}\right)=0, \quad \varphi^{(\mu)}\left(t_{0}\right) \neq 0,
$$

$\mu$ is called the order or multiplicity of $\varphi$ at $t=t_{0}$.

Definition A.1 ([14]). A linear subspace $\mathcal{A}$ of $\mathcal{C}^{\infty}(\boldsymbol{R})$ is called a Chebyshev space of order $n$ if it has the following:

(1) $\operatorname{dim} \mathcal{A} \geq n$.

(2) If $n$ is an odd number, each $\varphi \in \mathcal{A} \backslash\{0\}$ is $2 \pi$-periodic, i.e., $\varphi(t+2 \pi)=\varphi(t)$ for $t \in \boldsymbol{R}$. Moreover, $\varphi \in \mathcal{A}$ has at most $n-1$ zeros counted with multiplicities, when it is regarded as a function on $\boldsymbol{R} / 2 \pi \boldsymbol{Z}$. 
(3) If $n$ is an even number, each $\varphi \in \mathcal{A} \backslash\{0\}$ is $2 \pi$-odd-periodic, i.e., $\varphi(t+2 \pi)=-\varphi(t)$ for $t \in \boldsymbol{R}$. Moreover, $\varphi \in \mathcal{A}$ has at most $2(n-1)$ zeros counted with multiplicities, when it is regarded as a function on $\boldsymbol{R} / 4 \pi \boldsymbol{Z}$.

EXAMPLE A.2 (cf. [14]). For $N \in N$,

$$
\mathcal{A}_{2 N+1}:=\left\{\varphi \in \mathcal{C}^{\infty}(\boldsymbol{R}) ; \varphi(t)=a_{0}+\sum_{k=1}^{N}\left(a_{k} \cos k t+b_{k} \sin k t\right)\right\}
$$

is a Chebyshev space of order $2 N+1$. Similarly,

$$
\mathcal{A}_{2 N}:=\left\{\varphi \in \mathcal{C}^{\infty}(\boldsymbol{R}) ; \varphi(t)=\sum_{k=1}^{N}\left(a_{k} \cos \left(\frac{(2 k-1) t}{2}\right)+b_{k} \sin \left(\frac{(2 k-1) t}{2}\right)\right)\right\}
$$

is a Chebyshev space of order $2 N$.

The following lemma means that a Chebyshev space of order $n$ is isomorphic to $\boldsymbol{R}^{n}$. In particular, (1) of Definition A.1 can be replaced by the condition $\operatorname{dim} \mathcal{A}=n$.

LeMmA A.3 (cf. [4], [14]). Let $\mathcal{A} \subset \mathcal{C}^{\infty}(\boldsymbol{R})$ be a Chebyshev space of order $n$. Take $r(\leq n)$ arbitrary points $t_{1}, t_{2}, \ldots, t_{r}$ satisfying $0 \leq t_{1}<t_{2}<\cdots<t_{r}<2 \pi$ and $r$ positive integers $v_{1}, \ldots, v_{r}$ satisfying $v_{1}+\cdots+v_{r}=n$. Then, the linear map $T: \mathcal{A} \rightarrow \boldsymbol{R}^{n}$ defined by

$$
T(\varphi):=\left(j^{\nu_{1}-1} \varphi\left(t_{1}\right), j^{\nu_{2}-1} \varphi\left(t_{2}\right), \ldots, j^{\nu_{r}-1} \varphi\left(t_{r}\right)\right)
$$

is an isomorphism, where $j^{k} \varphi(t)$ is the $k$-jet of $\varphi$ of $t$, i.e.,

$$
j^{k} \varphi(t):=\left(\varphi(t), \varphi^{\prime}(t), \varphi^{\prime \prime}(t), \ldots, \varphi^{(k)}(t)\right)
$$

for each non-negative integer $k$.

Proof. We suppose that $\varphi \in \mathcal{A}$ satisfies $T(\varphi)=0$. Then, $\varphi$ has at least $n$ zeros with multiplicities unless $\varphi$ vanishes identically. This is a contradiction by the definition of Chebyshev spaces, so $\varphi=0$. Therefore, the linear map $T$ is injective. Moreover, $T$ is bijective by the condition $\operatorname{dim} \mathcal{A} \geq n$.

We consider a method to approximate a $C^{\infty}$-function by $C^{\omega}$-functions while fixing the jets at given points. The next lemma is used in the proof of Lemma 4.1 in order to control jets and to construct a required $C^{\omega}$-function.

LEMmA A.4. Let $\varphi(t)$ be a $2 \pi$-periodic $C^{\infty}$-function. Let $t_{1}, t_{2}, \ldots, t_{r}$ be $r$ points satisfying $0 \leq t_{1}<t_{2}<\cdots<t_{r}<2 \pi$ and $v_{1}, \ldots, v_{r}$ be positive integers. Then, there exists a sequence $\left\{\varphi_{q}\right\}_{q=1}^{\infty}$ of $2 \pi$-periodic $C^{\omega}$-functions such that

$$
j^{v_{i}-1} \varphi_{q}\left(t_{i}\right)=j^{v_{i}-1} \varphi\left(t_{i}\right) \quad(1 \leq i \leq r)
$$

for each $q \in N$ and $\left\{\varphi_{q}^{(k)}\right\}_{q=1}^{\infty}$ converges uniformly to $\varphi^{(k)}$ on $\boldsymbol{R}$ for each $k \in \boldsymbol{N}$.

PROOF. We may assume that the equality $v_{1}+\cdots+v_{r}=2 N+1$ holds for some $N \in N$ by replacing $v_{r}+1$ with $v_{r}$, if necessary. We set $\mathcal{A}:=\mathcal{A}_{2 N+1}$. The linear map 
$T: \mathcal{A} \rightarrow \boldsymbol{R}^{2 N+1}$ as in Lemma A.3 gives an isomorphism. A $2 \pi$-periodic $C^{\omega}$-function $b(t)$ is defined by $T(b)=\left(j^{v_{i}-1} \varphi\left(t_{i}\right)\right)_{1 \leq i \leq r}$. Then, there exists a $2 \pi$-odd-periodic $C^{\infty}$-function $\psi(t)$ such that

$$
\varphi(t)=b(t)+\left(\prod_{i=1}^{r} \sin ^{v_{i}}\left(\frac{t-t_{i}}{2}\right)\right) \psi(t) .
$$

Actually, for each $i=1, \ldots, r$, we have the equalities

$$
\lim _{t \rightarrow t_{i}}(\varphi-b)^{(k)}(t)=\lim _{t \rightarrow t_{i}} \frac{d^{k}}{d t^{k}}\left(\sin ^{v_{i}}\left(\frac{t-t_{i}}{2}\right)\right)=0 \quad\left(k=0,1, \ldots, v_{i}-1\right)
$$

and

$$
\lim _{t \rightarrow t_{i}} \frac{d^{v_{i}}}{d t^{v_{i}}}\left(\sin ^{v_{i}}\left(\frac{t-t_{i}}{2}\right)\right)=\frac{v_{i} !}{2^{v_{i}}} \neq 0 .
$$

Moreover, $\sin \left(\left(t_{l}-t_{i}\right) / 2\right)$ does not vanish if $l \neq i$, so $\psi(t)$ is even smooth at $t=t_{i}$. Then, there exists a sequence $\left\{\psi_{q}\right\}_{q=1}^{\infty}$ of $2 \pi$-odd-periodic $C^{\omega}$-functions such that $\left\{\psi_{q}^{(k)}\right\}_{q=1}^{\infty}$ converges uniformly to $\psi^{(k)}$ on $\boldsymbol{R}$ for each $k \in \boldsymbol{N}$. Therefore, we set

$$
\varphi_{q}(t)=b(t)+\left(\prod_{i=1}^{r} \sin ^{v_{i}}\left(\frac{t-t_{i}}{2}\right)\right) \psi_{q}(t),
$$

and then $\left\{\varphi_{q}^{(k)}\right\}_{q=1}^{\infty}$ converges uniformly to $\varphi^{(k)}$ on $\boldsymbol{R}$. Moreover, we have

$$
j^{v_{i}-1} \varphi_{q}\left(t_{i}\right)=j^{v_{i}-1} b\left(t_{i}\right)=j^{v_{i}-1} \varphi\left(t_{i}\right) \quad(1 \leq i \leq r)
$$

for each $q \in N$.

Acknowledgments. The author is very grateful to the referee and Jin-ichi Itoh for valuable comments, and to Atsufumi Honda for pointing out the importance of extrinsically flat surfaces. The author also thanks Masaaki Umehara and Kotaro Yamada for encouragement and suggestions, and Wayne Rossman for carefully reading and commenting to this paper.

\section{REFERENCES}

[ 1 ] J. A. Aledo, J. A. GÀlvez And P. Mira, A D'Alembert formula for flat surfaces in the 3-sphere, J. Geom. Anal. 19 (2009), 211-232.

[ 2 ] C. Chicone and N. J. Kalton, Flat embeddings of the Möbius strip in $\boldsymbol{R}^{3}$, Comm. Appl. Nonlinear Anal. 9 (2002), 31-50.

[ 3 ] J. A. GÀlvez And P. Mira, Isometric immersions of $\boldsymbol{R}^{2}$ into $\boldsymbol{R}^{4}$ and perturbation of Hopf tori, Math. Z. 266 (2010), 207-227.

[ 4 ] L. Guieu, E. Mourre and V. Yu Ovsienko, Theorem on six vertices of a plane curve via Sturm theory, The Arnold-Gelfand mathematical seminars, 257-266, Birkhäuser Boston, Boston, MA.

[ 5 ] A. HondA, Isometric immersions of the hyperbolic plane into the hyperbolic space, Tohoku Math. J. 64 (2012), 171-193.

[6] A. HONDA, Surfaces of constant Gaussian curvature-1 with singularities in the 3-sphere, preprint.

[7] Y. Kurono And M. Umehara, Flat Möbius strips of given isotopy type in $R^{3}$ whose centerlines are geodesics or lines of curvature, Geom. Dedicata 134 (2008), 109-130.

[ 8 ] M. Kokubu, W. Rossman, M. Umehara And K. Yamada, Flat fronts in hyperbolic 3-space and their caustics, J. Math. Soc. Japan 59 (2007), 265-299. 
[ 9 ] K. NAOKAWA, Singularities of the asymptotic completion of developable Möbius strips, to appear in Osaka J. Math.

[10] W. F. PoHL, The self-linking number of a closed space curve, J. Math. Mech. 17 (1968), 975-985.

[11] T. Randrup And P. RøGEn, Sides of the Möbius strip, Arch. Math. 66 (1996), 511-521.

[12] P. RøGEN, Embedding and knotting of flat compact surfaces in 3-space, Comment. Math. Helv. 76 (2001), 589-606.

[13] I. K. SABITOV, Isometric immersions and embeddings of locally Euclidean metrics, Cambridge Scientific Publishers, 2009.

[14] G. Thorbergsson And M. Umehara, A global theory of flexes of periodic functions, Nagoya Math. J. 173 (2004), 85-138.

[15] W. WunderLICH, Über ein abwickelbares Möbiusband, Monatsh. Math. 66 (1962), 276-289.

Department of Mathematics

TOKYO InSTITUTE OF TECHNOLOGY

2-12-1 OOKAYAMA

MEgURO-KU, TOKYO 152-8551

JAPAN

E-mail address: naokawa1@is.titech.ac.jp 